\title{
Long-Time Effect of Relaxation for Hyperbolic Conservation Laws ${ }^{\star}$
}

\section{I-Liang Chern}

Department of Mathematics, National Taiwan University, Taipei, Taiwan, R.O.C. Email address: chern@math.ntu.edu.tw

Received: 28 March 1994

\begin{abstract}
The hyperbolic conservation laws with relaxation appear in many physical systems such as nonequilibrium gas dynamics, flood flow with friction, viscoelasticity, magnetohydrodynamics, etc. This article studies the long-time effect of relaxation when the initial data is a perturbation of an equilibrium constant state. It is shown that in this case the long-time effect of relaxation is equivalent to a viscous effect, or in other words, the Chapman-Enskog expansion is valid. It is also shown that the corresponding solution tends to a diffusion wave time asymptotically. This diffusion wave carries an invariant mass. The convergence rate to this diffusion wave in the $L^{p}$-sense for $1 \leqq p \leqq \infty$ is also obtained and this rate is optimal.
\end{abstract}

\section{Introduction}

We consider the following model of hyperbolic conservation laws with relaxation $[5,8]$ :

$$
\begin{aligned}
u_{t}+f(u, v)_{x} & =0, \\
v_{t}+g(u, v)_{x} & =h(u, v), \quad-\infty<x<\infty, \quad t>0 .
\end{aligned}
$$

A concrete physical model is the following flood flow with friction:

$$
\begin{aligned}
h_{t}+(h u)_{x} & =0, \\
(h u)_{t}+\left(h u^{2}+\frac{g h^{2}}{2}\right)_{x} & =\left(g h \tan \alpha-C_{f} u^{2}\right),
\end{aligned}
$$

where $h$ is the height of the water, $u$, the velocity, $g$, the gravitation constant, $\alpha$, the inclined angle of the river, and $C_{f}$, the friction coefficient of the river. Other physical models include the nonequilibrium gas dynamics, the viscoelasticity, the magnetohydrodynamics, etc. [8].

\footnotetext{
$\star$ This work was supported by the National Science Council of the Republic of China under Contract NSC82-0208-M002-093
} 
System (1.1) (1.2) is assumed to be strictly hyperbolic, i.e. the matrix $\partial(f, g) / \partial(u, v)$ has two real and distinct eigenvalues $\lambda_{1}(u, v)<\lambda_{2}(u, v)$. The corresponding left/right eigenvectors are denoted by $l_{i}(u, v) / r_{i}(u, v), i=1,2$, respectively. We assume the relaxation term $h(u, v)$ to satisfy the following condition [5]: there is a smooth function $v_{*}(u)$ such that $h\left(u, v_{*}(u)\right)=0$ and

$$
\frac{\partial h(u, v)}{\partial v}<0
$$

for all $(u, v)$ under consideration. The states $\left(u, v_{*}(u)\right)$ are called the equilibrium states. Assumption (1.3) means that the equilibrium states are stable. To have nontrivial coupling of Eqs. (1.1) and (1.2), we further assume

$$
f_{v}(u, v) \neq 0 \text {. }
$$

System (1.1) (1.2) has been studied by Liu [5]. There, the nonlinear stability of weak, smooth travelling waves and rarefaction waves have been rigorously analyzed by the energy method. Liu also argued via the Chapman-Enskog expansion that the effect of relaxation is closely related to a viscous effect when the solution is near a constant equilibrium state. This article provides a rigorous proof to his argument. We show that the long-time effect of relaxation is equivalent to a viscous effect when the initial data is a perturbation of a constant equilibrium state. Further, we show that the corresponding solution tends to a diffusion wave [2] time asymptotically. This diffusion wave carries an invariant mass. The convergence rate to this diffusion wave is also obtained and this rate is optimal.

Without loss of generality, we may assume the constant state considered to be the zero state and we may further assume $v_{*}(0)=0$.

\section{Two Observations}

To study the effect of relaxation, we first see the following two observations.

2.1. Chapman-Enskog Expansion. When $v=v_{*}(u)$, system (1.1) and (1.2) are reduced to

$$
u_{t}+f_{*}(u)_{x}=0,
$$

where $f_{*}(u)=f\left(u, v_{*}(u)\right)$. This is called the equilibrium equation. When $v \neq v_{*}(u)$ but close, we expect that the solution of system (1.1) (1.2) propagates according to the equilibrium equation with a correction coming from the fluctuation $v_{1}=$ $v-v_{*}(u)$. To see this correction, we perform the following Chapman-Enskog expansion [5]. Let $v=v_{*}(u)+v_{1}$. Substitute this into (1.2) to obtain

$$
\begin{aligned}
v_{t}+g(u, v)_{x} & =h(u, v) \\
& \sim h_{v}\left(u, v_{*}(u)\right) v_{1} .
\end{aligned}
$$

This gives $v_{1} \sim h_{v}^{-1}\left(v_{t}+g_{x}\right)$. We further expand $v_{t}+g_{x}$ about $(0,0)$ and neglect all higher-order terms to obtain an expression of $v_{1}$ in terms of $u_{x}$ :

$$
\begin{aligned}
v_{1} & \sim h_{v}^{-1}\left(v_{t}+g(u, v)_{x}\right) \\
& \sim h_{v}^{-1}\left(v_{*}^{\prime} u_{t}+g_{u} u_{x}+g_{v} v_{*}^{\prime} u_{x}\right) \\
& \sim h_{v}^{-1}\left(-v_{*}^{\prime} \lambda_{*}+g_{u}+g_{v} v_{*}^{\prime}\right) u_{x} \\
& =-f_{v}^{-1} \frac{\left(\lambda_{*}-\lambda_{1}\right)\left(\lambda_{2}-\lambda_{*}\right)}{k} u_{x} .
\end{aligned}
$$


Here and after, the functions $h_{v}, g_{u}, \lambda_{i}$, etc. are evaluated at $(0,0), \lambda_{*} \equiv f_{*}^{\prime}(0)$, and $k$ stands for $h_{v}^{-1}$. In the derivation above we have used that $u_{t} \sim-\lambda_{*} u_{x}$. This is due to the assumption that $u$ propagates mainly along the equilibrium direction $d x / d t=\lambda_{*}$. Substitute this approximation of $v_{1}$ into $(1.1)$; we obtain

$$
\begin{aligned}
0 & =u_{t}+f\left(u, v_{*}(u)+v_{1}\right)_{x} \\
& \sim u_{t}+f_{*}(u)_{x}+\left(f_{v} v_{1}\right)_{x} \\
& \sim u_{t}+f_{*}(u)_{x}-v u_{x x}
\end{aligned}
$$

where

$$
v=\left(\lambda_{*}-\lambda_{1}\right)\left(\lambda_{2}-\lambda_{*}\right) / k .
$$

We notice that the reduced equation

$$
u_{t}+f_{*}(u)_{x}=v u_{x x}
$$

is well-posed for $v>0$. To have a nontrivial influence of fluctuation to system (1.1) and (1.2), it is natural to make the following stability assumption:

$$
v>0
$$

or equivalently,

$$
\lambda_{1}<\lambda_{*}<\lambda_{2} \text {. }
$$

Thus, the fluctuation $v_{1}$ produces a diffusion effect on the equilibrium equation under the stability assumption and the assumption that all higher-order nonlinear terms are less important.

2.2. Linear Analysis. We can take another viewpoint to see the effect of fluctuation. We linearize system (1.1) and (1.2) about $(0,0)$ to obtain

$$
\left(\begin{array}{l}
u \\
v
\end{array}\right)_{t}+\left(\begin{array}{ll}
f_{u} & f_{v} \\
g_{u} & g_{v}
\end{array}\right)\left(\begin{array}{l}
u \\
v
\end{array}\right)_{x}=\left(\begin{array}{cc}
0 & 0 \\
h_{u} & h_{v}
\end{array}\right)\left(\begin{array}{l}
u \\
v
\end{array}\right)
$$

Or equivalently,

$$
\left(\begin{array}{l}
u \\
v
\end{array}\right)_{t}=R\left(\frac{\partial}{\partial x}\right)\left(\begin{array}{l}
u \\
v
\end{array}\right)
$$

The solution operator $e^{t R}$ has the following Fourier representation:

$$
\frac{1}{2 \pi} \int e^{t R(i \xi)} e^{i x \xi} d \xi
$$

with

$$
R(i \xi)=-i \xi\left(\begin{array}{ll}
f_{u} & f_{v} \\
g_{u} & g_{v}
\end{array}\right)+\left(\begin{array}{cc}
0 & 0 \\
h_{u} & h_{v}
\end{array}\right) .
$$

The spectral representations of $R(i \xi)$ and $e^{t R(i \xi)}$ are

$$
\begin{aligned}
& R(i \xi)=\sum_{j=1}^{2} \mu_{j}(i \xi) P_{j}(i \xi) \\
& e^{t R(i \xi)}=\sum_{j=1}^{2} e^{t \mu_{j}(i \xi)} P_{j}(i \xi)
\end{aligned}
$$


respectively. Here, $\mu_{j}(i \xi)$ is the eigenvalues of $R(i \xi), P_{j}(i \xi)$, the projection oprerator to the corresponding right eigenvectors $r_{j}(i \xi)$, i.e. $P_{j} \equiv r_{j} \otimes l_{j}$, and $l_{j}$ is the left eigenvector. From a direct calculation and by using the stability assumption (2.4), we find that for any $\xi_{0}>0$, there exists a constant $\beta>0$ such that

$$
\operatorname{Re} \mu_{j}(i \xi) \leqq-\beta, j=1,2
$$

for $|\xi| \geqq \xi_{0}$. Thus, the fluctuation with frequency $|\xi| \geqq \xi_{0}$ decays exponentially. For low-frequency fluctuation, i.e. $|\xi|<<1, \mu_{j}(i \xi), j=1,2$ have the following asymptotic expansion:

$$
\begin{aligned}
\mu_{1}(i \xi) & =-i \lambda_{*} \xi-v \xi^{2}+O\left(|\xi|^{3}\right) \\
& \equiv \mu_{1}^{0}(i \xi)+O\left(|\xi|^{3}\right) \\
\mu_{2}(i \xi) & =-k-i \xi\left(\lambda_{1}+\lambda_{2}-\lambda_{*}\right)+O\left(|\xi|^{2}\right) \\
& \equiv \mu_{2}^{0}(i \xi)+O\left(|\xi|^{2}\right) .
\end{aligned}
$$

Formula (2.9) indicates that low-frequency fluctuation in the $r_{2}$ direction decays exponentially. However, in the $r_{1}$ direction, the Fourier inverse of $e^{t \mu_{1}^{0}(i \xi)}$ is the kernel of the following diffusion equation:

$$
u_{t}+\lambda_{*} u_{x}=v u_{x x}
$$

which in turn decays only algebraically. Thus, the long-time, long-range effect of relaxation is equivalent to a viscous effect in the linear sense.

From the above two observations, we expect the Chapman-Enskog expansion to be valid in the sense of large time. This means that system (1.1) (1.2) and Eq. (2.3) are expected to be equivalent time-asymptotically.

\section{Main Theorem}

It has been shown in $[1,2,4]$ that the time-asymptotic solution of $(2.3)$ is a diffusion wave, which is the self-similar solution of the following Burgers equation carrying a constant mass $m=\int u_{0}(x) d x$ :

$$
\begin{gathered}
\theta_{t}+\lambda_{*} \theta_{x}+\left(\frac{1}{2} f_{*}^{\prime \prime}(0) \theta^{2}\right)_{x}=v \theta_{x x}, \\
\theta(x,-1)=m \delta(x) .
\end{gathered}
$$

This diffusion wave has the following exact expression:

$$
\begin{aligned}
\theta(x, t) & =\frac{1}{\sqrt{t+1}} \theta^{*}\left(\frac{x-\lambda_{*}(t+1)}{\sqrt{t+1}}\right), \\
\theta^{*}(\xi) & = \begin{cases}\frac{m}{4 \pi v} e^{-\frac{\xi^{2}}{4 v}} & \text { if } f_{*}^{\prime \prime}(0)=0, \\
-\frac{1}{b}(\ln \psi(\xi))^{\prime} & \text { if } b=f_{*}^{\prime \prime}(0) \neq 0,\end{cases} \\
\psi(\xi) & =e^{b m / 4} \int_{-\infty}^{\xi / \sqrt{v}} e^{-y^{2} / 4} d y+e^{-b m / 4} \int_{\xi / \sqrt{v}}^{\infty} e^{-y^{2} / 4} d y .
\end{aligned}
$$


Since $\int u-\theta d x=0$, we may let $u=\theta+z_{x}$. By a simple calculation, it is natural to let $v=v_{*}(\theta)-f_{v}^{-1} \theta_{x}+w$. We substitute them into (1.1) and (1.2) to obtain

$$
\begin{aligned}
z_{t}+f_{u} z_{x}+f_{v} w & =\tilde{H}_{1}, \\
w_{t}+g_{u} z_{x x}+g_{v} w_{x} & =h_{u} z_{x}+h_{v} w+\tilde{H}_{2},
\end{aligned}
$$

where

$$
\begin{gathered}
\tilde{H}_{1}=\tilde{H}_{1}^{1}+\tilde{H}_{1}^{2}, \\
\tilde{H}_{2}=\tilde{H}_{2}^{1}+\tilde{H}_{2}^{2}+H_{2 x}, \\
\tilde{H}_{1}^{1}=O\left(|\theta|^{3}+\left|\theta \theta_{x}\right|+\theta_{x}^{2}\right), \\
\tilde{H}_{2}^{1}=O\left(|\theta|^{3}+\left|\theta \theta_{x}\right|+\theta_{x}^{2}+\left|\theta_{x x}\right|+\left|\theta_{x t}\right|+\left|\theta \theta_{t}\right|\right), \\
\tilde{H}_{1}^{2}, \tilde{H}_{2}^{2}, H_{2}=O\left(|\theta|+\left|z_{x}\right|+|w|\right)\left(\left|z_{x}\right|+|w|\right) .
\end{gathered}
$$

We shall measure the initial perturbation by

$$
\delta \equiv m+\sum_{l=0}^{3}\left\|D_{x}^{l} z(0)\right\|+\sum_{l=0}^{2}\left\|D_{x}^{l} w(0)\right\|+\|z(0)\|_{L^{1}}+\|w(0)\|_{L^{1}} .
$$

Here, $\|\cdot\|$ is the abbreviation of $\|\cdot\|_{L^{2}}$, and $z(t)$, the abbreviation of $z(\cdot, t)$. Our main theorem is stated as follows.

Theorem 1. Suppose the initial perturbation $\delta \ll 1$, then there exists a unique global smooth solution $(u, v)$ of $(1.1)$ and (1.2). Further, let $\theta$ be defined by (3.1), then the solution converges to the equilibrium diffusion wave $\left(\theta, v_{*}(\theta)\right)$ time asymptotically with the following rate estimates: for $l=0,1,2$,

$$
\begin{array}{r}
\left\|D_{x}^{l}[u(t)-\theta(t)]\right\|_{L^{p}}=O(1+t)^{-1-\frac{l}{2}+\frac{1}{2 p}}, \quad 1 \leqq p \leqq \infty, \\
\left\|D_{x}^{l}\left[v(t)-v_{*}(\theta(t))\right]\right\|_{L^{p}}=O(1+t)^{-1-\frac{l}{2}+\frac{1}{2 p}}, \quad 2 \leqq p \leqq \infty .
\end{array}
$$

The proof of Theorem 1 follows easily from an a priori decay estimate of $z$ and $w$ in the $L^{2}$-sense, which is the main task of this article. Thus, we shall prove Theorem 1 at the end of this article. To describe such an a priori estimate, we define the following notations: given any $T>0$, define

$$
\begin{aligned}
\||z|\|_{T} & \equiv \sup _{0 \leqq t \leqq T} \sum_{l=0}^{3}(1+t)^{1 / 4+l / 2}\left\|D_{x}^{l} z(t)\right\|, \\
\|\mid w\|_{T} & \equiv \sup _{0 \leqq t \leqq T} \sum_{l=0}^{2}(1+t)^{3 / 4+l / 2}\left\|D_{x}^{l} w(t)\right\|,
\end{aligned}
$$

and

$$
\varepsilon(T) \equiv \sup _{0 \leqq t \leqq T} \sum_{l=0}^{1}\left(\left|D_{x}^{l} \theta(t)\right|_{\infty}+\left|D_{x}^{l+1} z(t)\right|_{\infty}+\left|D_{x}^{l} w(t)\right|_{\infty}\right),
$$

where $|\cdot|_{\infty}$ stands for the sup-norm. Our main task is to prove the following a priori estimate. 
Proposition 1 (A priori estimate). If $\varepsilon(T)$ of (3.10) and $\delta$ of (3.7) are small, then

$$
\left\||| z\left|\left\|_{T}+\right\|\right| w \mid\right\|_{T} \leqq C
$$

for some constant $C$ which is independent of $T$.

We shall break the proof of Proposition 1 into the following two steps:

1. energy and decay estimates for the linearized equations,

2. energy and decay estimates for the nonlinear equations.

\section{Proof of the A Priori Estimate}

4.1. Decay Estimate for the Linearized Equations. In this section, we study the decay of solutions of the linearized equations

$$
\begin{aligned}
z_{t}+f_{u} z_{x}+f_{v} w & =0, \\
w_{t}+g_{u} z_{x x}+g_{v} w_{x} & =h_{u} z_{x}+h_{v} w .
\end{aligned}
$$

Proposition 2 (Energy estimate). The solution $(z(t), w(t))$ of the linear equations (4.1) and (4.2) has the following energy estimate: given any $t>0$, any integer $l \geqq 0$, we have

$$
\begin{gathered}
\left\|D_{x}^{l} z(t)\right\|^{2}+\left\|D_{x}^{l+1} z(t)\right\|^{2}+\left\|D_{x}^{l} w(t)\right\|^{2}+\int_{0}^{t}\left\|D_{x}^{l+1} z(s)\right\|^{2}+\left\|D_{x}^{l} w(s)\right\|^{2} d s \\
\leqq C\left(\left\|D_{x}^{l} z(0)\right\|^{2}+\left\|D_{x}^{l+1} z(0)\right\|^{2}+\left\|D_{x}^{l} w(0)\right\|^{2}\right)
\end{gathered}
$$

for some positive constant $C$ independent of $t$.

Proof. Substituting $w$ of (4.1) into (4.2), we obtain the telegraph equation:

$$
z_{t t}+\left(\lambda_{1}+\lambda_{2}\right) z_{x t}+\lambda_{1} \lambda_{2} z_{x x}+k\left(z_{t}+\lambda_{*} z_{x}\right)=0 .
$$

Changing the variable to $\xi=x-\lambda_{*} t$ and using (2.2) we obtain

$$
z_{t t}+\left(\lambda_{1}+\lambda_{2}-2 \lambda_{*}\right) z_{\xi t}-k v z_{\xi \xi}+k z_{t}=0
$$

$\int(4.4) z d \xi$ gives

$$
\frac{d}{d t}\left(\int z_{t} z+\frac{k}{2}\|z\|^{2}\right)+k v\left\|z_{\xi}\right\|^{2}-\left\|z_{t}\right\|^{2}=\hat{\lambda} \int z_{t} z_{\xi},
$$

where $\hat{\lambda}=\lambda_{1}+\lambda_{2}-2 \lambda_{*}$. Applying the Cauchy-Schwartz inequality on $\int z_{t} z_{\xi}$, we obtain

$$
\frac{k}{2} \frac{d}{d t}\|z\|^{2}+\frac{k v}{2}\left\|z_{\xi}\right\|^{2}-\left(1+\frac{2 \hat{\lambda}^{2}}{k v}\right)\left\|z_{t}\right\|^{2} \leqq-\frac{d}{d t} \int z_{t} z .
$$

$\int(4.4) z_{t} d \xi$ gives

$$
\frac{d}{d t}\left(\frac{1}{2}\left\|z_{t}\right\|^{2}+\frac{k v}{2}\left\|z_{\xi}\right\|^{2}\right)+k\left\|z_{t}\right\|^{2}=0
$$


We choose $\gamma>0$ to satisfy

$$
\left(1+\frac{2 \hat{\lambda}^{2}}{k v}\right) \gamma \leqq \frac{k}{2}
$$

Then $\gamma(4.5)+(4.6)$ gives

$$
\frac{d}{d t}\left(\frac{k \gamma}{2}\|z\|^{2}+\frac{1}{2}\left\|z_{t}\right\|^{2}+\frac{k v}{2}\left\|z_{\xi}\right\|^{2}\right)+\frac{k}{2}\left\|z_{t}\right\|^{2}+\frac{k v \gamma}{2}\left\|z_{\xi}\right\|^{2} \leqq-\gamma \frac{d}{d t} \int z_{t} z .
$$

We integrate this equation in $t$ from 0 to $t$ and apply the Cauchy-Schwartz inequality to handle $\int z_{t} z$. In this process, we require $\gamma$ to further satisfy

$$
\frac{4 \gamma}{k} \leqq \frac{1}{4}
$$

then we obtain

$$
\begin{aligned}
& \frac{k \gamma}{4}\|z(t)\|^{2}+\frac{1}{4}\left\|z_{t}(t)\right\|^{2}+\frac{k v}{2}\left\|z_{\xi}(t)\right\|^{2}+\int_{0}^{t}\left(\frac{k}{2}\left\|z_{t}(s)\right\|^{2}+\frac{k v \gamma}{2}\left\|z_{\xi}(s)\right\|^{2}\right) d s \\
& \leqq C\left(\|z(0)\|^{2}+\left\|z_{t}(0)\right\|^{2}+\left\|z_{\xi}(0)\right\|^{2}\right) .
\end{aligned}
$$

We can change the variable $\xi$ back to $x$ to obtain

$$
\begin{aligned}
\|z(t)\|^{2}+\left\|z_{x}(t)\right\|^{2} & +\left\|z_{t}(t)\right\|^{2}+\int_{0}^{t}\left\|z_{x}(s)\right\|^{2}+\left\|z_{t}(s)\right\|^{2} d s \\
& \leqq C\left(\|z(0)\|^{2}+\left\|z_{x}(0)\right\|^{2}+\left\|z_{t}(0)\right\|^{2}\right) .
\end{aligned}
$$

Here, we have used that

$$
\left\|\left.\partial_{t}\right|_{x} z\right\|^{2}-C_{1}\left\|z_{x}\right\|^{2} \leqq C_{2}\left\|\left.\partial_{t}\right|_{\xi} z\right\|^{2} \leqq\left\|\left.\partial_{t}\right|_{x} z\right\|^{2}+C_{1}\left\|z_{x}\right\|^{2}
$$

for some positive constants $C_{1}$ and $C_{2}$. This is followed from chain rule. Similarly, we also have from (4.1) that

$$
\|w(t)\|^{2}-C_{1}\left\|z_{x}(t)\right\|^{2} \leqq C_{2}\left\|z_{t}(t)\right\|^{2} \leqq\|w(t)\|^{2}+C_{1}\left\|z_{x}(t)\right\|^{2} .
$$

Thus, we can further convert $\left\|z_{t}\right\|$ of (4.10) in terms of $\|w\|$ and $\left\|z_{x}\right\|$ to obtain

$$
\begin{aligned}
\|z(t)\|^{2}+\left\|z_{x}(t)\right\|^{2} & +\|w(t)\|^{2}+\int_{0}^{t}\left\|z_{x}(s)\right\|^{2}+\|w(s)\|^{2} d s \\
& \leqq C\left(\|z(0)\|^{2}+\left\|z_{x}(0)\right\|^{2}+\|w(0)\|^{2}\right) .
\end{aligned}
$$

This proves the lemma for $l=0$. Notice that $\left(D_{x}^{l} z, D_{x}^{l} w\right)$ satisfies the same equation, therefore (4.3) also holds for $l>0$.

Proposition 3. Let $(z(t), w(t))$ be the solution of (4.1) and (4.2) with initial data $(z(0), w(0))$. Then there exist constants $\beta>0, C>0$ such that

$$
\begin{aligned}
\|z(t)\|^{2} \leqq & C e^{-2 \beta t}\left(\left\|D_{x} z(0)\right\|^{2}+\|z(0)\|^{2}+\|w(0)\|^{2}\right) \\
& +C(1+t)^{-1 / 2}\left(\|z(0)\|_{L^{1}}^{2}+\|w(0)\|_{L^{1}}^{2}\right)
\end{aligned}
$$


and

$$
\begin{aligned}
\left\|D_{x}^{l} z(t)\right\|^{2},\left\|D_{x}^{l-1} w(t)\right\|^{2} \leqq & C e^{-2 \beta t}\left(\left\|D_{x}^{l} z(0)\right\|^{2}+\left\|D_{x}^{l-1} z(0)\right\|^{2}+\left\|D_{x}^{l-1} w(0)\right\|^{2}\right) \\
& +C(1+t)^{-1 / 2-l+k}\left(\left\|D_{x}^{k} z(0)\right\|_{L^{1}}^{2}+\left\|D_{x}^{k} w(0)\right\|_{L^{1}}^{2}\right)
\end{aligned}
$$

for $l \geqq 1,0 \leqq k \leqq l-1$. Here, the constant $C$ is independent of $t$.

Proof. The solution $(z(t), w(t))$ of (4.1) and (4.2) has the following Fourier representation:

$$
\left(\begin{array}{c}
i \xi \hat{z}(\xi, t) \\
\hat{w}(\xi, t)
\end{array}\right)=e^{t R(i \xi)}\left(\begin{array}{c}
i \xi \hat{z}(\xi, 0) \\
\hat{w}(\xi, 0)
\end{array}\right),
$$

where $R(i \xi)$ is given by (2.5). We use (4.13) and the Parseval equality to estimate $\left\|D_{x}^{l} z(t)\right\|^{2}$ and $\left\|D_{x}^{l-1} w(t)\right\|^{2}$. In the integration in the Fourier space, we break the integral $\int d \xi$ into $\int_{|\xi|<\xi_{0}} d \xi+\int_{|\xi|>\xi_{0}} d \xi$, where $\xi_{0}$ is a small positive number to be chosen later. For $|\xi|>\xi_{0}$, we have from (2.6), (2.7), (4.13) and the Parseval equality that there is a positive constant $\beta$ such that

$$
\int_{|\xi|>\xi_{0}}\left|(i \xi)^{l} \hat{z}(\xi, t)\right|^{2}+\left|(i \xi)^{l-1} \hat{w}(\xi, t)\right|^{2} d \xi \leqq C e^{-2 \beta t}\left(\left\|D_{x}^{l} z(0)\right\|^{2}+\left\|D_{x}^{l-1} w(0)\right\|^{2}\right) .
$$

For $|\xi|<\xi_{0}, l_{j}(i \xi)$ and $r_{j}(i \xi)$ have the following asymptotic expression:

$$
\begin{aligned}
& l_{1}(i \xi)=(1,0)+O(|\xi|), \\
& l_{2}(i \xi)=\left(-v_{*}^{\prime}(0), 1\right)+O(|\xi|), \\
& r_{1}(i \xi)=\left(\begin{array}{c}
1 \\
v_{*}^{\prime}(0)
\end{array}\right)+O(|\xi|), \\
& r_{2}(i \xi)=\left(\begin{array}{c}
0 \\
1
\end{array}\right)+O(|\xi|) .
\end{aligned}
$$

We denote the dominating terms of $l_{j}(i \xi) / r_{j}(i \xi)$ by $l_{j}^{0} / r_{j}^{0}$, respectively and define $P_{j}^{0} \equiv r_{j}^{0} \otimes l_{j}^{0}$. We then choose $\xi_{0}$ so small that when $|\xi| \leqq \xi_{0}$ we have

$$
\operatorname{Re}(-v+O(|\xi|))<-\alpha \text { and } \operatorname{Re}(-k+O(|\xi|))<-\beta
$$

for some constants $\alpha>0$ and $\beta>0$. Then from (2.8) and (2.9) we have

$$
e^{t R(i \xi)}=\sum_{j=1}^{2} e^{t \mu_{J}^{0}(i \xi)} P_{j}^{0}(i \xi)+O(|\xi|) e^{-\alpha \xi^{2} t}+O\left(e^{-\beta t}\right)
$$

for $|\xi|<\xi_{0}$. Now, from (2.8), (2.9), (4.13) and (4.14) we have

$$
\begin{aligned}
\int_{|\xi|<\xi_{0}}\left|(i \xi)^{l} \hat{z}(\xi, t)\right|^{2} d \xi \leqq & \int_{|\xi|<\xi_{0}}\left|e^{u_{1}^{0}(i \xi) t}(i \xi)^{l} \hat{z}(\xi, 0)\right|^{2} d \xi \\
& +\int_{|\xi|<\xi_{0}} C e^{-2 \alpha \xi^{2} t}\left(\left|\xi^{l+1} \hat{z}(\xi, 0)\right|^{2}+\left|\xi^{l} \hat{w}(\xi, 0)\right|^{2}\right) d \xi \\
& +\int_{|\xi|<\xi_{0}} C e^{-2 \beta t}\left(\left|\xi^{l} \hat{z}(\xi, 0)\right|^{2}+\left|\xi^{l-1} \hat{w}(\xi, 0)\right|^{2}\right) d \xi \\
= & I+I I+I I I .
\end{aligned}
$$


Here, we have chosen $\beta \leqq k$ and absorbed the term $e^{\mu_{2}^{0}(i \xi) t}$ into $e^{-\beta t}$. We extend the domain of integration from $|\xi|<\xi_{0}$ to the whole line. Then

$$
\begin{aligned}
I & \leqq\left\|G(t) * D_{x}^{l} z(0)\right\|^{2} \\
& \leqq C t^{-1 / 2-l+k}\left\|D_{x}^{k} z(0)\right\|_{L^{1}}^{2}, \\
I I & \leqq C t^{-3 / 2-l+k}\left\|D_{x}^{k} z(0)\right\|_{L^{1}}^{2}+C t^{-1 / 2-l+k}\left\|D_{x}^{k} w(0)\right\|_{L^{1}}^{2}, \\
I I I & \leqq C e^{-2 \beta t}\left(\left\|D_{x}^{l} z(0)\right\|^{2}+\left\|D_{x}^{l-1} w(0)\right\|^{2}\right) .
\end{aligned}
$$

Here, $G(x, t)$ is the heat kernel $\frac{1}{\sqrt{4 \pi v t}} e^{\left(x-\lambda_{*} t\right)^{2} /(4 v t)}$. Similarly,

$$
\begin{aligned}
\int_{|\xi|<\xi^{0}}\left|(i \xi)^{l-1} \hat{w}(\xi, t)\right|^{2} d \xi \leqq & \int_{|\xi|<\xi^{0}}\left|e^{\mu_{1}^{0}(i \xi) t} i \xi^{l} v_{*}^{\prime} \hat{z}(\xi, 0)\right|^{2} \\
& +C e^{-2 \alpha \xi^{2} t}\left(\left|\xi^{l+1} \hat{z}(\xi, 0)\right|^{2}+\left|\xi^{l} \hat{w}(\xi, 0)\right|^{2}\right) \\
& +C e^{-2 \beta t}\left(\left|\xi^{l} \hat{z}(\xi, 0)\right|^{2}+\left|\xi^{l-1} \hat{w}(\xi, 0)\right|^{2}\right) \\
= & I+I I+I I I .
\end{aligned}
$$

The terms $I, I I$ and $I I I$ have the same estimate as above. Summarizing the above estimations, we obtain

$$
\begin{aligned}
\left\|D_{x}^{l} z(t)\right\|^{2},\left\|D_{x}^{l-1} w(t)\right\|^{2} \leqq & C e^{-2 \beta t}\left(\left\|D_{x}^{l} z(0)\right\|^{2}+\left\|D_{x}^{l-1} w(0)\right\|^{2}\right) \\
& +C t^{-1 / 2-l+k}\left(\left\|D_{x}^{k} z(0)\right\|_{L^{1}}^{2}+\left\|D_{x}^{k} w(0)\right\|_{L^{1}}^{2}\right) \\
& +C t^{-3 / 2-l+k}\left\|D_{x}^{k} z(0)\right\|_{L^{1}}^{2} .
\end{aligned}
$$

This formula together with the energy estimate (4.3) gives (4.12). Next, we prove (4.11). We represent $(z(t), w(t))$ by

$$
\left(\begin{array}{c}
\hat{z}(\xi, t) \\
\hat{w}(\xi, t)
\end{array}\right)=e^{t \tilde{R}(i \xi)}\left(\begin{array}{c}
\hat{z}(\xi, 0) \\
\hat{w}(\xi, 0)
\end{array}\right)
$$

where

$$
\tilde{R}(i \xi)=-\left(\begin{array}{cc}
i \xi f_{u} & f_{v} \\
(i \xi)^{2} g_{u} & i \xi g_{v}
\end{array}\right)+\left(\begin{array}{cc}
0 & 0 \\
i \xi h_{u} & h_{v}
\end{array}\right)
$$

Notice that $\tilde{R}(i \xi)=N^{-1}(i \xi) R(i \xi) N(i \xi)$, where $N(i \xi)=\left(\begin{array}{cc}i \xi & 0 \\ 0 & 1\end{array}\right)$. Therefore, $R(i \xi)$ and $\tilde{R}(i \xi)$ have the same eigenvalues. From (2.7) and (4.15) we have

$$
\int_{|\xi|>\xi_{0}}\left(|\hat{z}(\xi, t)|^{2}+|\hat{w}(\xi, t)|^{2}\right) d \xi \leqq C e^{-2 \beta t}\left(\|z(0)\|^{2}+\|w(0)\|^{2}\right) .
$$

For $|\xi|<\xi_{0}$, the eigenvectors of $\tilde{R}(i \xi)$ have the following asymptotic expansion:

$$
\begin{aligned}
& \tilde{l}_{1}(i \xi)=\left(1,-h_{v}^{-1} f_{v}\right)+O(|\xi|), \\
& \tilde{l}_{2}(i \xi)=(0,1)+O(|\xi|), \\
& \tilde{r}_{1}(i \xi)=\left(\begin{array}{c}
1 \\
0
\end{array}\right)+O(|\xi|), \\
& \tilde{r}_{2}(i \xi)=\left(\begin{array}{c}
h_{v}^{-1} f_{v} \\
1
\end{array}\right)+O(|\xi|) .
\end{aligned}
$$


By a similar calculation as above we obtain

$$
\begin{aligned}
\|z(t)\|^{2} \leqq & C e^{-2 \beta t}\left(\|z(0)\|^{2}+\|w(0)\|^{2}\right) \\
& +C\left(t^{-1 / 2}+t^{-3 / 2}\right)\left(\|z(0)\|_{L^{1}}^{2}+\|w(0)\|_{L^{1}}^{2}\right) .
\end{aligned}
$$

These formulae together with the energy estimate (4.3) gives (4.11).

4.2. Energy Estimate for the Nonlinear Equations. In this section, we give an a priori estimate for the solution of the nonlinear equations (3.2) and (3.3). Let us assume the solution $(z(t), w(t))$ of (3.2) and (3.3) to exist in $[0, T]$, where $T>0$ is an arbitrary number.

Proposition 4. Let us assume the solution $(z(t), w(t))$ of (3.2) and (3.3) to exist in $[0, T]$ and assume $\varepsilon(T)$ of (3.10) and $\delta$ of (3.7) to be small. Then there exist constant $\beta>0, C>0$, which are independent of $T$, such that we have for $t \in$ $[0, T]$,

$$
\begin{aligned}
& \left\|D_{x}^{l} z(t)\right\|^{2}+\left\|D_{x}^{l+1} z(t)\right\|^{2}+\left\|D_{x}^{l} w(t)\right\|^{2} \\
& \quad+\int_{0}^{t} e^{-2 \beta(t-s)}\left(\left\|D_{x}^{l+1} z(s)\right\|^{2}+\left\|D_{x}^{l} w(s)\right\|^{2}\right) d s \\
& \leqq C e^{-2 \beta t}\left(\left\|D_{x}^{l} z(0)\right\|^{2}+\left\|D_{x}^{l+1} z(0)\right\|^{2}+\left\|D_{x}^{l} w(0)\right\|^{2}\right) \\
& \quad+C \int_{0}^{t} e^{-2 \beta(t-s)}\left(\left\|D_{x}^{l} \theta(s)\right\|^{2}+\left\|D_{x}^{l} z(s)\right\|^{2}+\left\|D_{x}^{l-1} w(s)\right\|^{2}\right) d s .
\end{aligned}
$$

Proof. When $\varepsilon(T)$ is small, we can solve $w$ from (3.3) in terms of $z_{t}$ and $z_{x}$ :

$$
w=-f_{v}^{-1}\left(z_{t}+f_{u} z_{x}\right)+f_{v}^{-1} H_{1}^{1}+O\left(|\theta|+\left|z_{t}\right|+\left|z_{x}\right|\right) z_{t}+O\left(|\theta|+\left|z_{t}\right|+\left|z_{x}\right|\right) z_{x} .
$$

Substituting this into (3.2) and changing the variable from $x$ to $\xi=x-\lambda_{*} t$, we obtain

$$
z_{t t}+\left(\lambda_{1}+\lambda_{2}-2 \lambda_{*}\right) z_{t \xi}-k v z_{\xi \xi}-z_{t}=K
$$

where

$$
\begin{aligned}
K & =K_{1}+K_{2}+K_{3 t}+K_{4 \xi}, \\
K_{1} & =\tilde{H}_{1}^{1}-f_{v} \tilde{H}_{2}^{1}, \\
K_{i} & =A_{t} z_{t}+B_{i} z_{\xi}, i=2,3,4, \\
A_{i}, B_{i} & =O\left(|\theta|+\left|z_{t}\right|+\left|z_{\xi}\right|\right), i=2,3,4 .
\end{aligned}
$$

Let $\nabla$ denote for $\left(\partial_{t}, \partial_{\xi}\right)$ and let us define

$$
\rho(T) \equiv \sup _{0 \leqq t \leqq T} \sum_{l=0}^{1}\left(\left|\nabla^{l} \theta(t)\right|_{\infty}+\left|\nabla^{l+1} z(t)\right|_{\infty}\right) .
$$


We claim that the statement of this proposition is equivalent to the following statement:

$$
\begin{aligned}
& \left\|\nabla^{l} z(t)\right\|^{2}+\left\|\nabla^{l+1} z(t)\right\|^{2}+\int_{0}^{t} e^{-2 \beta(t-s)}\left\|\nabla^{l+1} z(s)\right\|^{2} d s \\
& \leqq C e^{-2 \beta t}\left(\left\|\nabla^{l} z(0)\right\|^{2}+\left\|\nabla^{l+1} z(0)\right\|^{2}\right) \\
& \quad+C \int_{0}^{t} e^{-2 \beta(t-s)}\left(\left\|\nabla^{l} \theta\right\|^{2}+\left\|\nabla^{l} z\right\|^{2}\right) d s,
\end{aligned}
$$

provided $\rho(T)$ and $\delta$ are sufficiently small. The proof of this claim is basically followed by the following lemma.

Lemma 1. There exist constants $C_{i}, i=1,2,3,4$ which are independent of $T$ such that

$$
\begin{aligned}
C_{1} \varepsilon(T) & \leqq \rho(T) \leqq C_{2} \varepsilon(T), \\
C_{1}\left\|D_{x}^{l} \theta\right\| & \leqq\left\|\nabla^{l} \theta\right\| \leqq C_{2}\left\|D_{x}^{l} \theta\right\|, \\
\left\|D_{x}^{l} w(t)\right\|+\left\|D_{x}^{l+1} z(t)\right\| & \leqq C_{3}\left(\left\|\nabla^{l+1} z(t)\right\|+\varepsilon(T)\left\|\nabla^{l} \theta(t)\right\|\right)
\end{aligned}
$$

and

$$
\left\|\nabla^{l+1} z(t)\right\| \leqq C_{4}\left(\left\|D_{x}^{l} w(t)\right\|+\left\|D_{x}^{l+1} z(t)\right\|+\rho(T)\left\|D_{x}^{l} \theta(t)\right\|\right)
$$

for $0 \leqq t \leqq T$, provided $\varepsilon(T) \ll 1$ or $\rho(T) \ll 1$.

This lemma can be proved directly by (3.2), (4.17) and Lemmas A1 and A2. We omit the details.

Now, we perform the energy estimate for (4.18) as follows. From Lemma 1, we may assume $\rho(T)$ to be small. Let us abbreviate it by $\rho$. Let $\gamma$ be the constant satisfying (4.7) and (4.8). Then $\int \nabla^{l}(4.18) \cdot\left(\gamma \nabla^{l} z+\nabla^{l} z_{t}\right) d \xi$ gives

$$
\begin{aligned}
\frac{d}{d t}\left(\frac{k \gamma}{2}\left\|\nabla^{l} z\right\|^{2}\right. & \left.+\frac{1}{2}\left\|\nabla^{l} z_{t}\right\|^{2}+\frac{k v}{2}\left\|\nabla^{l} z_{\xi}\right\|^{2}\right)+\frac{k}{2}\left\|\nabla^{l} z_{t}\right\|^{2}+\frac{k v \gamma}{2}\left\|\nabla^{l} z_{\xi}\right\|^{2} \\
& \leqq-\gamma \frac{d}{d t} \int \nabla^{l} z_{t} \cdot \nabla^{l} z d \xi+\int \nabla^{l} K \cdot\left(\gamma \nabla^{l} z+\nabla^{l} z_{t}\right) d \xi
\end{aligned}
$$

Further, $\int_{0}^{t} e^{-2 \beta(t-s)}(4.22) d s$ for any $\beta \geqq 0$ gives

$$
\begin{aligned}
& \frac{k \gamma}{2}\left\|\nabla^{l} z(t)\right\|^{2}+\frac{1}{2}\left\|\nabla^{l} z_{t}(t)\right\|^{2}+\frac{k v}{2}\left\|\nabla^{l} z_{\xi}(t)\right\|^{2} \\
& \quad+\int_{0}^{t} e^{-2 \beta(t-s)}\left(-\beta k \gamma\left\|\nabla^{l} z(s)\right\|^{2}+\left(\frac{k}{2}-\beta\right)\left\|\nabla^{l} z_{t}(s)\right\|^{2}\right. \\
& \left.\quad+\left(\frac{k v \gamma}{2}-\beta k v\right)\left\|\nabla^{l} z_{\xi}(s)\right\|^{2}\right) d s
\end{aligned}
$$




$$
\begin{aligned}
\leqq & \gamma e^{-2 \beta t} \int\left|\nabla^{l} z_{t}(0) \cdot \nabla^{l} z(0)\right| d \xi+\gamma \int\left|\nabla^{l} z_{t}(t) \cdot \nabla^{l} z(t)\right| d \xi \\
& +2 \beta \gamma \int_{0}^{t} \int e^{-2 \beta(t-s)}\left|\nabla^{l} z_{t}(s) \cdot \nabla^{l} z(s)\right| d \xi d s \\
& +\int_{0}^{t} \int e^{-2 \beta(t-s)}\left|\nabla^{l} K \cdot\left(\gamma \nabla^{l} z+\nabla^{l} z_{t}\right)\right| d \xi d s . \\
\leqq & C e^{-2 \beta t}\left(\left\|\nabla^{l} z(0)\right\|^{2}+\left\|\nabla^{l+1} z(0)\right\|^{2}\right) \\
& +\frac{k \gamma}{4}\left\|\nabla^{l} z(t)\right\|^{2}+\frac{4 \gamma}{k}\left\|\nabla^{l} z_{t}(t)\right\|^{2}+2 \beta \gamma \int_{0}^{t} e^{-2 \beta(t-s)}\left(\left\|\nabla^{l} z_{t}(s)\right\|^{2}+\left\|\nabla^{l} z(s)\right\|^{2}\right) d s \\
& +\int_{0}^{t} \int e^{-2 \beta(t-s)}\left|\nabla^{l} K \cdot\left(\gamma \nabla^{l} z+\nabla^{l} z_{t}\right)\right| d \xi d s .
\end{aligned}
$$

Here, we have used integration-by-part and the Cauchy-Schwartz inequality. Now, we require $\gamma$ to satisfy $(4.7),(4.8)$ and $\gamma \leqq \frac{1}{4}$. Further, we choose $\beta$ to satisfy

$$
\beta-2 \beta \gamma \leqq \frac{k}{4}
$$

and

Then we obtain

$$
\beta \leqq \frac{\gamma}{4}
$$

$$
\begin{aligned}
& \frac{k \gamma}{4}\left\|\nabla^{l} z(t)\right\|^{2}+\frac{1}{4}\left\|\nabla^{l} z_{t}(t)\right\|^{2}+\frac{k v}{2}\left\|\nabla^{l} z_{\xi}(t)\right\|^{2} \\
& \quad+\int_{0}^{t} e^{-2 \beta(t-s)}\left(\frac{k}{4}\left\|\nabla^{l} z_{t}(s)\right\|^{2}+\frac{k v \gamma}{4}\left\|\nabla^{l} z_{\xi}(s)\right\|^{2}\right) d s \\
& \leqq C e^{-2 \beta t}\left(\left\|\nabla^{l} z(0)\right\|^{2}+\left\|\nabla^{l+1} z(0)\right\|^{2}\right)+C \int_{0}^{t} e^{-2 \beta(t-s)}\left\|\nabla^{l} z(s)\right\|^{2} d s \\
& \quad+\int_{0}^{t} \int e^{-2 \beta(t-s)}\left|\nabla^{l} K \cdot\left(\gamma \nabla^{l} z+\nabla^{l} z_{t}\right)\right| d \xi d s .
\end{aligned}
$$

We claim that the last term on the right-hand-side of (4.23) has the following estimate:

$$
\begin{aligned}
& \int_{0}^{t} \int e^{-2 \beta(t-s)}\left|\nabla^{l} K \cdot\left(\gamma \nabla^{l} z+\nabla^{l} z_{t}\right)\right| d \xi d s \\
& \leqq \\
& \quad C \rho\left(\left\|\nabla^{l} z(t)\right\|^{2}+\left\|\nabla^{l+1} z(t)\right\|^{2}\right) \\
& \quad+\int_{0}^{t} e^{-2 \beta(t-s)}\left(\frac{k}{8}\left\|\nabla^{l} z_{t}(s)\right\|^{2}+C(\rho+\beta)\left\|\nabla^{l+1} z(s)\right\|^{2}\right) d s \\
& \quad+C e^{-2 \beta t}\left(\left\|\nabla^{l} z(0)\right\|^{2}+\left\|\nabla^{l+1} z(0)\right\|^{2}\right) \\
& \quad+C \int_{0}^{t} e^{-2 \beta(t-s)}\left(\left\|\nabla^{l} \theta(s)\right\|^{2}+\left\|\nabla^{l} z(s)\right\|^{2}\right) d s .
\end{aligned}
$$

Once this claim is proved, we can choose $\beta$ to be small and adjust $\rho$ to be small enough so that the first two terms on the right-hand-side of (4.24) can be absorbed into the left-hand-side of (4.23). Thus, (4.21) is proved. Hence, it remains to prove 
(4.24). We need the following lemma.

Lemma 2. Let $A_{i}, B_{i}, i=2,3,4$ be defined by (4.19). Then

$$
\begin{aligned}
\left\|\nabla^{l} A_{i}\right\|,\left\|\nabla^{l} B_{i}\right\| & \leqq C\left(\left\|\nabla^{l} \theta\right\|+\left\|\nabla^{l+1} z\right\|\right) \\
\left|A_{i}\right|_{\infty},\left|B_{i}\right|_{\infty} & \leqq C \rho
\end{aligned}
$$

Also, we have

$$
\left\|\nabla^{l+1} \theta\right\| \leqq C\left\|\nabla^{l} \theta\right\|
$$

where $C$ is constant independent of $T$.

The proof of this lemma follows from Lemmas A1, A2 and (3.1) directly.

Now, we prove (4.24). We recall that $K=K_{1}+K_{2}+K_{3 t}+K_{4 \xi}$. From Lemma 2,

$$
\left\|\nabla^{l} K_{1}\right\| \leqq C\left\|\nabla^{l} \theta\right\|
$$

and

$$
\begin{aligned}
\left\|\nabla^{l} K_{2}\right\| & \leqq\left\|\nabla^{l} A_{2}\right\|\left|z_{t}\right|_{\infty}+\left\|\nabla^{l} B_{2}\right\|\left|z_{\xi}\right|_{\infty}+\left|A_{2}\right|_{\infty}\left\|\nabla^{l} z_{t}\right\|+\left|B_{2}\right|_{\infty}\left\|\nabla^{l} z_{\xi}\right\| \\
& \leqq C \rho\left(\left\|\nabla^{l} \theta\right\|+\left\|\nabla^{l+1} z\right\|\right) .
\end{aligned}
$$

Hence,

$$
\begin{aligned}
\int \nabla^{l} K_{1} \cdot\left(\gamma \nabla^{l} z+\nabla^{l} z_{t}\right) \mid d \xi & \leqq C\left\|\nabla^{l} K_{1}\right\|\left(\left\|\nabla^{l} z\right\|+\left\|\nabla^{l} z_{t}\right\|\right) \\
& \leqq \frac{k}{8}\left\|\nabla^{l} z_{t}\right\|^{2}+C\left(\left\|\nabla^{l} \theta\right\|^{2}+\left\|\nabla^{l} z\right\|^{2}\right), \\
\int\left|\nabla^{l} K_{2} \cdot\left(\gamma \nabla^{l} z+\nabla^{l} z_{t}\right)\right| d \xi & \leqq C\left\|\nabla^{l} K_{2}\right\|\left(\left\|\nabla^{l} z\right\|+\left\|\nabla^{l} z_{t}\right\|\right) \\
& \leqq C \rho\left(\left\|\nabla^{l} \theta\right\|^{2}+\left\|\nabla^{l} z\right\|^{2}+\left\|\nabla^{l+1} z\right\|^{2}\right) .
\end{aligned}
$$

Next, we estimate $\int\left|\nabla^{l}\left(\partial_{t} K_{3}+\partial_{\xi} K_{4}\right) \cdot\left(\gamma \nabla^{l} z+\nabla^{l} z_{t}\right)\right| d \xi$. We recall that $K_{i}=$ $A_{i} z_{t}+B_{i} z_{\xi}, i=3,4$. We shall only compute the term $\int \nabla^{l} \partial_{t}\left(A_{3} z_{t}\right) \cdot \nabla^{l} z d \xi$ in detail. The rest terms can be handled similarly. We have

$$
\nabla^{l} \partial_{t}\left(A_{3} z_{t}\right)=A_{3}^{\prime} \nabla^{l} z_{t t}+A_{3}^{\prime \prime} \nabla^{l} z_{t \xi}+\text { L.O.T. }
$$

where $A_{3}^{\prime}, A_{3}^{\prime \prime}$ satisfy (4.19), and L.O.T. are the lower order terms. By Lemmas 2, $\mathrm{A} 1$ and $\mathrm{A} 2$, the L.O.T. have the following estimate:

$$
\begin{aligned}
\| \text { L.O.T. } \| & \leqq C\left(\left\|\nabla^{l} \theta\right\|+\left\|\nabla^{l+1} z\right\|\right)\left(\left|z_{t}\right|_{\infty}+\left|z_{t t}\right|_{\infty}\right)+\left|A_{3, t}\right|_{\infty}\left\|\nabla^{l} z_{t}\right\| \\
& \leqq C \rho\left(\left\|\nabla^{l} \theta\right\|+\left\|\nabla^{l+1} z\right\|\right) \\
\int A_{3}^{\prime} \nabla^{l} z_{t t} \cdot \nabla^{l} z d \xi & =\frac{d}{d t} \int A_{3}^{\prime} \nabla^{l} z_{t} \cdot \nabla^{l} z-\int A_{3 t}^{\prime} \nabla^{l} z_{t} \cdot \nabla^{l} z-\int A_{3}^{\prime}\left(\nabla^{l} z_{t}\right)^{2} \\
& \leqq \frac{d}{d t} \int A_{3}^{\prime} \nabla^{l} z_{t} \cdot \nabla^{l} z+C \rho\left\|\nabla^{l} z_{t}\right\|\left(\left\|\nabla^{l} z\right\|+\left\|\nabla^{l} z_{t}\right\|\right)
\end{aligned}
$$




$$
\begin{aligned}
\int A_{3}^{\prime \prime} \nabla^{l} z_{t \xi} \cdot \nabla^{l} z & \leqq \frac{d}{d t} \int A_{3}^{\prime \prime} \nabla^{l} z_{\xi} \cdot \nabla^{l} z+O(\rho)\left\|\nabla^{l} z_{\xi}\right\|\left(\left\|\nabla^{l} z_{t}\right\|+\left\|\nabla^{l} z\right\|\right) \\
\int A_{3}^{\prime} \nabla^{l} z_{t t} \cdot \nabla^{l} z_{t} & =\frac{1}{2} \frac{d}{d t} \int A_{3}^{\prime}\left(\nabla^{l} z_{t}\right)^{2}-\frac{1}{2} \int A_{3 t}^{\prime}\left(\nabla^{l} z_{t}\right)^{2} \\
& \leqq \frac{1}{2} \frac{d}{d t} \int A_{3}^{\prime}\left(\nabla^{l} z_{t}\right)^{2}+C \rho\left\|\nabla^{l} z_{t}\right\|^{2} \\
\int A_{3}^{\prime \prime} \nabla^{l} z_{t \xi} \cdot \nabla^{l} z_{t} & =-\frac{1}{2} \int A_{3 \xi}^{\prime \prime}\left(\nabla^{l} z_{t}\right)^{2}=O(\rho)\left\|\nabla^{l} z_{t}\right\|^{2} .
\end{aligned}
$$

After computing $\int\left|\nabla^{l} \partial_{t}\left(B_{3} z_{\xi}\right) \cdot \nabla^{l} z\right| d \xi, \cdots, \int \nabla^{l} \partial_{\xi}\left(B_{4} z_{\xi}\right) \cdot \nabla^{l} z_{t} d \xi$ by a similar way, we reach

$$
\begin{aligned}
& \int\left|\nabla^{l}\left(\partial_{t} K_{3}+\partial_{\xi} K_{4}\right) \cdot\left(\gamma \nabla^{l} z+\nabla^{l} z_{t}\right)\right| d \xi \\
& \leqq \\
& C \rho\left(\left\|\nabla^{l} \theta\right\|^{2}+\left\|\nabla^{l} z\right\|^{2}+\left\|\nabla^{l+1} z\right\|^{2}\right) \\
& \quad+\frac{d}{d t} \int\left(\tilde{A} \nabla^{l} z_{\xi} \cdot \nabla^{l} z+\tilde{B} \nabla^{l} z_{t} \cdot \nabla^{l} z+\tilde{C}\left(\nabla^{l} z_{t}\right)^{2}+\tilde{D} \nabla^{l} z_{t} \cdot \nabla^{l} z_{\xi}+\tilde{E}\left(\nabla^{l} z_{\xi}\right)^{2}\right) d \xi,
\end{aligned}
$$

where $\tilde{A}, \cdots, \tilde{E}$ satisfy (4.19). Combining (4.25), (4.26) and (4.27), we obtain

$$
\begin{aligned}
& \int\left|\nabla^{l} K \cdot\left(\gamma \nabla^{l} z+\nabla^{l} z_{t}\right)\right| d \xi \\
& \leqq \\
& \frac{k}{8}\left\|\nabla^{l} z_{t}\right\|^{2}+C \rho\left\|\nabla^{l+1} z\right\|^{2}+C\left(\left\|\nabla^{l} \theta\right\|^{2}+\left\|\nabla^{l} z\right\|^{2}\right) \\
& \quad+\frac{d}{d t} \int\left(\tilde{A} \nabla^{l} z_{\xi} \cdot \nabla^{l} z+\tilde{B} \nabla^{l} z_{t} \cdot \nabla^{l} z+\tilde{C}\left(\nabla^{l} z_{t}\right)^{2}+\tilde{D} \nabla^{l} z_{t} \cdot \nabla^{l} z_{\xi}+\tilde{E}\left(\nabla^{l} z_{\xi}\right)^{2}\right) d \xi .
\end{aligned}
$$

Finally, $\int_{0}^{t} e^{-2 \beta(t-s)}(4.28) d s$ and using integration-by-part and that $|\tilde{A}|_{\infty}, \cdots,|\tilde{E}|_{\infty}$ $=O(\rho)$, we obtain (4.24).

4.3. Decay Estimate for the Nonlinear Equations. In this section, we give the a priori decay estimate for the solution of the nonlinear equations (3.2) and (3.3). We need the following two lemmas [2].

Lemma 3. The diffusion wave $\theta$ has the following estimate:

$$
\left\|D_{x}^{l} \theta(t)\right\|_{L^{p}}=O(\delta)(1+t)^{-\frac{1}{2}-\frac{l}{2}+\frac{1}{2 p}}, l \geqq 0 .
$$

The proof of this lemma follows easily from the exact expression of $\theta$.

Lemma 4. Let $\alpha, \beta, \gamma$ be positive numbers. Then

$$
\int_{0}^{t} e^{-\beta(t-s)}(1+s)^{-\gamma} d s=O(1+t)^{-\alpha}
$$

if $\alpha \leqq \gamma$,

$$
\int_{0}^{t / 2}(1+t-s)^{-\beta}(1+s)^{-\gamma} d s=O(1+t)^{-\alpha}
$$

if $\alpha \leqq \beta, \alpha \leqq \beta+\gamma-1, \gamma \neq 1$, or if $\alpha<\beta, \alpha \leqq \beta+\gamma-1, \gamma=1$,

$$
\int_{t / 2}^{t}(1+t-s)^{-\beta}(1+s)^{-\gamma} d s=O(1+t)^{-\alpha}
$$

if $\alpha \leqq \gamma, \alpha \leqq \beta+\gamma-1, \gamma \neq 1$, or if $\alpha<\gamma, \alpha \leqq \beta+\gamma-1, \beta=1$. 
Proof of Proposition 1. Let us assume the solution $(z(t), w(t))$ of (3.2) and (3.3) exists in $[0, T], T>0$ and $\varepsilon(T) \ll 1$. We use the following integral representation to estimate $\left\|D_{x}^{l} z(t)\right\|$ and $\left\|D_{x}^{l-1} w(t)\right\|$ :

$$
\begin{aligned}
\left(\begin{array}{c}
z(t) \\
w(t)
\end{array}\right) & =e^{t \tilde{R}\left(\partial_{x}\right)}\left(\begin{array}{c}
z(0) \\
w(0)
\end{array}\right)+\int_{0}^{t} e^{(t-s) \tilde{R}\left(\partial_{x}\right)}\left(\begin{array}{c}
\tilde{H}_{1}(s) \\
\tilde{H}_{2}(s)
\end{array}\right) d s \\
& =\left(\begin{array}{c}
z^{0}(t) \\
w^{0}(t)
\end{array}\right)+\left(\begin{array}{c}
\tilde{z}(t) \\
\tilde{w}(t)
\end{array}\right) .
\end{aligned}
$$

From Proposition 3, we have

$$
\begin{gathered}
\left\|D_{x}^{l} z^{0}(t)\right\|^{2}=O(1+t)^{-1 / 2-l} \delta, \quad l \geqq 0, \\
\left\|D_{x}^{l-1} w^{0}(t)\right\|^{2}=O(1+t)^{-1 / 2-l} \delta, \quad l \geqq 1, \\
\|\tilde{z}(t)\|^{2} \leqq C \int_{0}^{t} e^{-2 \beta(t-s)}\left(\left\|H_{1}\right\|^{2}+\left\|D_{x} H_{2}\right\|^{2}\right) d s \\
+C \int_{0}^{t}(1+t-s)^{-1 / 2}\left(\left\|H_{1}\right\|_{L^{1}}^{2}+\left\|D_{x} H_{2}\right\|_{L^{1}}^{2}\right),
\end{gathered}
$$

and for $l \geqq 1$,

$$
\begin{aligned}
\left\|D_{x}^{l} \tilde{z}(t)\right\|^{2}, & \left\|D_{x}^{l-1} \tilde{w}(t)\right\|^{2} \leqq C \int_{0}^{t} e^{-2 \beta(t-s)}\left(\left\|D_{x}^{l-1} H_{1}\right\|^{2}+\left\|D_{x}^{l} H_{1}\right\|^{2}+\left\|D_{x}^{l} H_{2}\right\|^{2}\right) d s \\
& +C \int_{0}^{t / 2}(1+t-s)^{-1 / 2-l}\left\|H_{1}\right\|_{L^{1}}^{2}+C \int_{0}^{t / 2}(1+t-s)^{-3 / 2-l}\left\|H_{2}\right\|_{L^{1}}^{2} \\
& +C \int_{t / 2}^{t}(1+t-s)^{-3 / 2}\left\|D_{x}^{l-1} H_{1}\right\|_{L^{1}}^{2}+C \int_{t / 2}^{t}(1+t-s)^{-5 / 2}\left\|D_{x}^{l-1} H_{2}\right\|_{L^{1}}^{2} .
\end{aligned}
$$

Here,

$$
\begin{aligned}
H_{1}= & \tilde{H}_{1}^{1}+\tilde{H}_{1}^{2}+\tilde{H}_{2}^{1}+\tilde{H}_{2}^{2} \\
= & O\left(|\theta|^{3}+\left|\theta \theta_{x}\right|+\left|\theta_{x x}\right|+\theta_{x}^{2}+\left|\theta_{x t}\right|+\left|\left(\theta^{2}\right)_{t}\right|\right) \\
& +O\left(|\theta|+\left|z_{x}\right|+|w|\right)\left(\left|z_{x}\right|+|w|\right), \\
H_{2}= & O\left(|\theta|+\left|z_{x}\right|+|w|\right)\left(\left|z_{x}\right|+|w|\right) .
\end{aligned}
$$

Let us abbreviate $\||z|||+|||w|||$ by $M$. By using (4.35), (4.36), (3.8) and (3.9), and applying Lemmas 3, A1 and A2, we obtain

$$
\begin{aligned}
\left\|D_{x}^{l} H_{1}\right\| & \leqq O(\delta)(1+s)^{-5 / 4-l / 2}+O(\delta+M)\left(\left\|D_{x}^{l+1} z\right\|+\left\|D_{x}^{l} w\right\|\right), \\
\left\|D_{x}^{l} H_{2}\right\| & \leqq O(\delta+M)\left(\left\|D_{x}^{l+1} z\right\|+\left\|D_{x}^{l} w\right\|\right), \\
\left\|D_{x}^{l-1} H_{1}\right\| & \leqq O(1+s)^{-3 / 4-l / 2}(\delta+M(\delta+M)), \\
\left\|D_{x}^{l-1} H_{1}\right\|_{L^{1}} & \leqq O(1+s)^{-1 / 2-l / 2}(\delta+M(\delta+M)), \\
\left\|D_{x}^{l-1} H_{2}\right\|_{L^{1}} & \leqq O(1+s)^{-1 / 2-l / 2} M(\delta+M), \\
\left\|H_{1}\right\|_{L^{1}} & \leqq O(1+s)^{-1}(\delta+M(\delta+M)), \\
\left\|H_{2}\right\|_{L^{1}} & \leqq O(1+s)^{-1}(M(\delta+M)) .
\end{aligned}
$$


We apply the energy estimate to handle the term $\left\|D_{x}^{l+1} z\right\|^{2}+\left\|D_{x}^{l} w\right\|^{2}$ :

$$
\begin{aligned}
& \int_{0}^{t} e^{-2 \beta(t-s)}\left(\left\|D_{x}^{l+1} z(s)\right\|^{2}+\left\|D_{x}^{l} w(s)\right\|^{2}\right) d s \\
& \quad \leqq \int_{0}^{t} e^{-2 \beta(t-s)}\left(\left\|D_{x}^{l} \theta(s)\right\|^{2}+\left\|D_{x}^{l} z(s)\right\|^{2}+\left\|D_{x}^{l-1} w(s)\right\|^{2}\right) d s \\
& \quad \leqq C(1+t)^{-1 / 2-l}(\delta+M)^{2} .
\end{aligned}
$$

By (4.33), (4.34) Lemma 4, and the estimations of $H_{i}$ above, we obtain

$$
\begin{aligned}
\left\|D_{x}^{l} \tilde{z}(t)\right\|^{2} & \leqq C(1+t)^{-1 / 2-l}(\delta+M(\delta+M))^{2}, 0 \leqq l \leqq 3, \\
\left\|D_{x}^{l-1} \tilde{w}(t)\right\|^{2} & \leqq C(1+t)^{-1 / 2-l}(\delta+M(\delta+M))^{2}, 1 \leqq l \leqq 3 .
\end{aligned}
$$

From (3.8) and (3.9), we obtain

$$
M^{2} \leqq C(\delta+(\delta+M) M)^{2} .
$$

Thus, we have

$$
M \leqq C
$$

for some constant $C$ which is independent of $T$, provided $\delta$ and $\varepsilon(T)$ are small enough. This completes the proof of Proposition 1.

Proof of the Main Theorem. The local existence and uniqueness of the smooth solution of (3.2) and (3.3) are followed from the energy estimate with $\beta=0$. The global existence is followed from the local existence and the a priori decay estimate. The $L^{p}$-decay rates of $z$ and $w$ are obtained by interpolation. These proofs are standard. For reference, see [3]. Finally, the convergent rate obtained in Theorem 1 is the same as the optimal convergent rate to diffusion waves for scalar conservation laws [2]. Thus, this rate is optimal.

\section{Appendix}

We list two well-known lemmas which are used in the paper. For reference, see [6].

Lemma A1. If $u, v \in H^{l} \cap L^{\infty}$, then

$$
\begin{aligned}
\left\|D_{x}^{l}(u v)\right\| & \leqq\left(|u|_{\infty}\left\|D_{x}^{l} v\right\|+|v|_{\infty}\left\|D_{x}^{l} u\right\|\right), \\
\left\|D_{x}^{l}(u v)\right\|_{L^{1}} & \leqq\left(\|u\|\left\|D_{x}^{l} v\right\|+\|v\|\left\|D_{x}^{l} u\right\|\right) .
\end{aligned}
$$

Lemma A2. Suppose $N$ is smooth function in the neighborhood of 0 and $v \in$ $H^{l} \cap L^{\infty}$. Then there is a constant $\eta$ depending on $N$ and constant $C(\eta)$ such that

$$
\left\|D_{x}^{l} N(v)\right\| \leqq C(\eta)\left\|D_{x}^{l} v\right\|
$$

provided $|v|_{\infty} \leqq \eta$ 


\section{References}

1. Chern, I-L.: Multiple-mode diffusion waves for viscous nonstrictly hyperbolic conservation laws. Commun. Math. Phys. 138, 51-61 (1991)

2. Chern, I-L., Liu, Tai-Ping: Convergence to diffusion waves of solutions for viscous conservation laws. Commun. Math. Phys. 110, 503-517 (1987) 120, 525-527 (1989)

3. Kawashima, S.: Systems of a hyperbolic-parabolic composite type, with application to the equation of magnetohydrodynamics. Doctoral thesis, Kyoto University (1983)

4. Kawashima, S.: Large-time behavior of solutions to hyperbolic-parabolic systems of conservation laws and applications. Proc. R. Soc. Edinburgh 106 A, 169-194 (1987)

5. Liu, T.-P.: Hyperbolic conservation laws with relaxation. Commun. Math. Phys. 108, 153-175 (1987)

6. Moser, J.: A rapidly convergent iteration method and nonlinear partial differential equation. I. Ann. Sc. Norm. Super. Pisa 20, 265-315 (1966)

7. Vincenti, W., Kruger, C.: Introduction to physical gas dynamics. Melbourne: Robert E. Krieger, 1982

8. Whitham, J.: "Linear and nonlinear waves." New York: Wiley, 1974

Communicated by S.-T. Yau 
